\title{
Vilkovisky unique effective action in quantum gravity
}

\author{
Breno L. Giacchini $\odot,{ }^{1, *}$ Tibério de Paula Netto $\odot,{ }^{1, \dagger}$ and Ilya L. Shapiro $\circledast^{2, \ddagger}$ \\ ${ }^{1}$ Department of Physics, Southern University of Science and Technology, Shenzhen 518055, China \\ ${ }^{2}$ Departamento de Física, ICE, Universidade Federal de Juiz de Fora,
} Juiz de Fora, 36036-900 Minas Gerais, Brazil

(Received 13 September 2020; accepted 1 October 2020; published 5 November 2020)

\begin{abstract}
The divergent part of the one-loop Vilkovisky unique effective action for quantum Einstein gravity is evaluated in the general parametrization of the quantum field, including the separated conformal factor. The output of this calculation explicitly demonstrates the parametrization and conformal gauge independence of the unique effective action with the configuration space metric chosen following Vilkovisky's prescription.
\end{abstract}

DOI: 10.1103/PhysRevD.102.106006

\section{INTRODUCTION}

The off-shell effective action in gauge theories depends on the choice of the gauge fixing and the parametrization of quantum fields. On the other hand, in the modified versions of effective action proposed by Vilkovisky [1] and DeWitt [2], there is no gauge or parametrization ambiguity. The purpose of the present work is to evaluate the divergent part of the one-loop Vilkovisky effective action for the quantum version of Einstein gravity in a general parametrization of the quantum field and explicitly verify the independence of this construction on the parametrization.

The classical action of the theory of our interest has the form

$$
S\left(g_{\mu \nu}\right)=-\frac{1}{\kappa^{2}} \int \mathrm{d}^{D} x \sqrt{|g|}(R+2 \Lambda),
$$

where $G=\kappa^{2} /(16 \pi)$ is the (D-dimensional) Newton constant and $\Lambda$ is the cosmological constant. There is an extensive amount of literature on the derivation and analysis of one-loop and two-loop divergences in the theory (1). The first calculations were performed in Ref. [3] for gravity coupled with the minimal scalar field and in Ref. [4] for gravity coupled to an electromagnetic field. The calculation in the nonminimal gauge was pioneered in Ref. [5]. The parametrization dependence was explored in Refs. [6-8] and, in a more general form, in

\footnotetext{
*breno@sustech.edu.cn

tiberio@sustech.edu.cn

¥On leave from Tomsk State Pedagogical University; ilyashapiro2003@ufjf.br.

Published by the American Physical Society under the terms of the Creative Commons Attribution 4.0 International license. Further distribution of this work must maintain attribution to the author(s) and the published article's title, journal citation, and DOI. Funded by SCOAP ${ }^{3}$.
}

the more recent Ref. [9]. In what follows, we shall use some technical developments of the latter work, which can be also consulted for further references.

The unique effective action of Vilkovisky is independent of the parametrization of quantum fields by construction. On the other hand, this construction becomes complicated in gauge theories, where one has to combine corrections compensating gauge and parametrization ambiguities. In this regard, a special case is the two-dimensional quantum gravity. It was noted in Ref. [10] that, in this particular example, the gauge and parametrization ambiguities mix in such a way that the unique effective action turns out to depend on the gauge fixing. The origin of this contradictory result is that the unique effective action depends on the choice of the metric in the configuration space, or the space of the quantum fields, in the background field formalism, as it was anticipated in the very first work [1]. In gravity, the configuration-space metric has one arbitrary parameter $a$, and it happens that in the $D=2$ covariant formulation of the metric-scalar theory (see, e.g., Refs. [11,12] for the review) this parameter depends on the gauge fixing because of the reduced number of the physical degrees of freedom [10]. As a result, the metric in the configuration space depends on the gauge-fixing parameters even if the bilinear form of the action acquires the simplest minimal form. The four-dimensional quantum gravity in the conformal parametrization has a seeming similarity with the mentioned $D=2$ case because the metric in the configuration space also depends on a gauge parameter, namely, the conformal gauge-fixing parameter $\lambda[6,9]$. Thus, one could suspect that some gauge or parametrization dependence in the offshell unique effective action may persist in this case, too.

Let us note that the Vilkovisky-DeWitt approach in quantum gravity opens the way for formulating the exact renormalization group flow for the cosmological and Newton constants and for the full set of higher-derivative terms which should be added to the Einstein-Hilbert action 
in the framework of effective field theory [13] (see also Refs. [14,15] for previous analysis of the renormalization group based on the unique effective action in quantum gravity). This makes the verification of the consistency of this approach in $D=4$ even more interesting.

The outline of the paper is as follows. Section II briefly reviews the formalism of Vilkovisky's effective action. The main objective of this section is to make the paper selfconsistent and to fix the notations. In Sec. III, we formulate the one-loop quantum gravity using the background field method in a general nonconformal parametrization of quantum field and a special minimal gauge. The metric in the space of the fields, the Christoffel symbols, and the improved bilinear form of the classical action are derived in Sec. IV. It is shown that the coefficients related to the parametrization nonlinearity are compensated by this correction. The corresponding one-loop divergences of the Vilkovisky effective action are computed, in the minimal DeWitt gauge, in Sec. V. In Sec. VI, the result is generalized to the most general, conformal parametrization of the quantum metric. Finally, in Sec. VII, we draw our conclusions.

In this paper, we adopt the condensed notations of Refs. [16,17].

\section{VILKOVISKY EFFECTIVE ACTION: A SHORT REVIEW}

Vilkovisky's proposal for defining a parametrizationindependent effective action [1] is based on the following observation: even though the classical action $S(\varphi)$ is a scalar in the space $\mathcal{M}$ of fields $\varphi^{i}$, the generating functional of vertex functions (effective action) is not a scalar functional of the corresponding mean fields. In the simplest, one-loop approximation, the effective action depends on the Hessian of the action, $S_{, i j}=\frac{\delta^{2} S}{\delta \varphi^{i} \delta \varphi^{\prime}}$, which does not transform as a tensor under field redefinitions $\varphi^{i}=\varphi^{i}\left(\varphi^{\prime j}\right)$.

To provide the scalar nature of the effective action, Ref. [1] introduced an affine structure compatible with the metric $G_{i j}$ in the space $\mathcal{M}$. For given two close points $\varphi^{i}$ and $\varphi^{\prime i}$, there exists a unique geodesic curve $x^{i}(\lambda) \subset \mathcal{M}$ with affine parameter $\lambda \in[0,1]$ connecting them, $x^{i}(0)=$ $\varphi^{i}$ and $x^{i}(1)=\varphi^{\prime i}$. Then, defining the two-point quantity $\sigma^{i}\left(\varphi^{\prime}, \varphi\right)=\left.\frac{d x^{i}(\lambda)}{d \lambda}\right|_{\lambda=1}$ (the tangent vector to the geodesic at $\varphi^{\prime i}$; see e.g., Refs. $\left.[16,18]\right)$, the modified definition of the effective action has the form

$\exp i \Gamma(\varphi)=\int \mathcal{D} \varphi^{\prime} \mu\left(\varphi^{\prime}\right) \exp \left\{i\left[S\left(\varphi^{\prime}\right)+\sigma^{i}\left(\varphi, \varphi^{\prime}\right) \Gamma_{, i}(\varphi)\right]\right\}$,

where $\mu\left(\varphi^{\prime}\right)$ is an invariant functional measure and the comma denotes functional differentiation with respect to $\varphi^{i}$. The effective action $\Gamma(\varphi)$ constructed in this way is a scalar under field reparametrizations because $\sigma^{i}\left(\varphi, \varphi^{\prime}\right)$ behaves as a vector with respect to $\varphi^{i}$ and as a scalar with regard to $\varphi^{\prime i}$.
A qualitatively similar construction can be done for gauge theories, to restore the off-shell gauge independence, given that the effective actions calculated in different gauges are connected by changes of variables (in general, in the form of a canonical transformation [19-21]). However, in this case, the prescription (2) cannot be used directly since it is necessary to factor out the gauge group $\mathcal{G}$ in the functional integral. Namely, one has to take into account the gauge orbits and define an affine connection in the configuration space $\mathcal{M} / \mathcal{G}$ of physical fields. For the sake of simplicity, we assume that the generators $R_{\alpha}^{i}$ of gauge transformations are linearly independent and their algebra is closed, $R_{\beta, j}^{i} R_{\alpha}^{j}-R_{\alpha, j}^{i} R_{\beta}^{j}=F_{\alpha \beta}^{\gamma} R_{\gamma}^{i}$, with the structure functions $F_{\alpha \beta}^{\gamma}$ being independent of the fields. Let the classical action be invariant under gauge transformations $\delta \varphi^{i}=R_{\alpha}^{i} \xi^{\alpha}$,

$$
\varepsilon_{i} R_{\alpha}^{i}=0, \quad \varepsilon_{i} \equiv S_{, i} .
$$

Given a metric $G_{i j}$ on $\mathcal{M}$, one can define the projection operator on $\mathcal{M} / \mathcal{G}[1,22]$,

$$
P_{j}^{i}=\delta_{j}^{i}-R_{\alpha}^{i} N^{\alpha \beta} R_{\beta}^{k} G_{k j},
$$

where $N^{\alpha \beta}$ is the inverse of the metric on $\mathcal{G}$,

$$
N_{\alpha \beta}=R_{\alpha}^{i} G_{i j} R_{\beta}^{j} .
$$

Then, the projected metric is

$$
G_{i j}^{\perp \perp} \equiv P_{i}^{k} G_{k l} P_{j}^{l}=G_{i j}-G_{i k} R_{\alpha}^{k} N^{\alpha \beta} R_{\beta}^{l} G_{l j} .
$$

The affine connection $\mathcal{T}_{i j}^{k}$ on the physical configuration space can then be obtained by requiring its compatibility with the metric $G_{i j}^{\perp \perp}$ i.e., $\nabla_{k} G_{i j}^{\perp \perp}=0$ (see, e.g., Refs. [23,24]). This yields [1]

$$
\mathcal{T}_{i j}^{k}=\Gamma_{i j}^{k}+T_{i j}^{k}
$$

which consists of the Christoffel symbol $\Gamma_{i j}^{k}$ calculated with the metric $G_{i j}$,

$$
\Gamma_{i j}^{k}=\frac{1}{2} G^{k l}\left(G_{i l, j}+G_{j l, i}-G_{i j, l}\right),
$$

and a nonlocal part $T_{i j}^{k}$ related to the gauge constraints on the connection,

$$
\begin{aligned}
T_{i j}^{k}= & -2 G_{(i \mid l} R_{\alpha}^{l} N^{\alpha \beta} \mathcal{D}_{\mid j)} R_{\beta}^{k} \\
& +G_{(i \mid l} R_{\alpha}^{l} N^{\alpha \beta} R_{\beta}^{m}\left(\mathcal{D}_{m} R_{\gamma}^{k}\right) N^{\gamma \delta} R_{\delta}^{n} G_{n \mid j)} .
\end{aligned}
$$

The parentheses in the indices represent symmetrization in the pair $(i, j)$, and $\mathcal{D}_{i}$ denotes the covariant derivative calculated with the Christoffel connection $\Gamma_{i j}^{k}$. The nonlocality of (9) is due to the fact that $N_{\alpha \beta}$ is a differential 
operator and thus its inverse $N^{\alpha \beta}$ is formally a Green's function. In addition to that, this procedure provides the measure $\mu(\varphi)$ of the Faddeev-Popov quantization; see, e.g., Refs. [25,26]. The effective action (2) constructed using the geodesic distance based on the connection $\mathcal{T}_{i j}^{k}$ is therefore reparametrization invariant, gauge invariant, and gauge independent. For this reason, this object is often called unique effective action. ${ }^{1}$

Performing the loop expansion of the Vilkovisky effective action (2), one gets

$\Gamma(\varphi)=S(\varphi)+\bar{\Gamma}^{(1)}(\varphi)+\bar{\Gamma}^{(2)}(\varphi)+\cdots, \quad \hbar=1$,

where the one-loop quantum contribution is given by [1]

$\bar{\Gamma}^{(1)}=\frac{i}{2} \operatorname{Tr} \ln G^{i k}\left(\mathcal{D}_{k} \mathcal{D}_{j} S-T_{k j}^{l} \varepsilon_{l}-\chi_{, k}^{\alpha} Y_{\alpha \beta} \chi_{, j}^{\beta}\right)-i \operatorname{Tr} \ln M_{\beta}^{\alpha}$.

As usual, in pure quantum gravity, we can use $\kappa$ as a loop expansion parameter, instead of $\hbar$. Here, $\chi^{\alpha}$ is a gauge condition introduced by the gauge-fixing action

$$
S_{\mathrm{GF}}=-\frac{1}{2} \chi^{\alpha} Y_{\alpha \beta} \chi^{\beta}
$$

$Y_{\alpha \beta}$ is a nondegenerate weight function (the $\chi^{\alpha}$-space metric), and $M_{\beta}^{\alpha}=\chi_{i}^{\alpha} R_{\beta}^{i}$ is the Faddeev-Popov ghost matrix. Comparing (11) to the loop expansion of the standard effective action, one notes that the second functional derivative of the classical action has been replaced by the second covariant variational derivative.

From the technical side, the computation of (11) is, in general, a very complicated task because of the nonlocalities of the term $T_{i j}^{k}$. For this reason, most of the evaluations found in the literature use some kind of DeWitt gauge [30], for which

$$
\chi_{, i}^{\alpha}=-Y^{\alpha \beta} G_{i j} R_{\beta}^{j} .
$$

The following observation is in order. It is quite common in the literature (see, e.g., Refs. [22,24,27]) to use the singular version of (13), $\chi_{, i}^{\alpha}=0$, also known as Landau-DeWitt gauge. Such a gauge choice is convenient as it yields $T_{i j}^{k}=0$. Thus, in theories whose field space $\mathcal{M}$ is flat, at one-loop level, the traditional effective action evaluated in the Landau-DeWitt gauge is equal to Vilkovisky's one [22]. This gauge, however, is not so auspicious in gravity

\footnotetext{
${ }^{1}$ Another gauge- and parametrization-invariant effective action was proposed by DeWitt [2]. Since both definitions coincide at the one-loop level, we do not present this construction. We remark, however, that for calculations in higher-loop orders it is necessary to use the Vilkovisky-DeWitt formalism, as the simplest form (2) may generate nonlocal divergences [27,28] (see also Ref. [29]).
}

theories because the geometry of $\mathcal{M}$ is nontrivial $[22,27,29]$. A remarkable exception is the one-loop divergences related to the cosmological constant and EinsteinHilbert term in quantum general relativity. In fact, it turns out that for the Vilkovisky's choice of metric $G_{i j}$ in the space of fields the $\Gamma_{i j}^{k}$-correction in Eq. (11) does not give any new contribution to these terms; therefore, they can be directly obtained by using the Landau-DeWitt gauge in the context of the usual definition of the effective action [22]. As here we are interested in evaluating also the divergences related to curvature-squared terms, for practical reasons, we choose to use the nonsingular version of the DeWitt gauge and deal with the nonlocalities in the connection.

The purpose of the present work is to evaluate the divergent part of (11) for the quantum gravity based on the general relativity. In this calculation, we follow the reduction method introduced in Ref. [17], which mainly consists in making a power series expansion in the equations of motion $\varepsilon_{i}$ and applying the generalized Schwinger-DeWitt technique. By using the DeWitt gauge (13) and the Ward identities, it is possible to write (11) in the form [17]

$$
\begin{aligned}
\bar{\Gamma}^{(1)}= & \frac{i}{2} \operatorname{Tr} \ln \hat{H}-i \operatorname{Tr} \ln \hat{N} \\
& -\frac{i}{2}\left(\operatorname{Tr} \hat{U}_{1}-\operatorname{Tr} \hat{U}_{2}\right)-\frac{i}{4} \operatorname{Tr} \hat{U}_{1}^{2}+O\left(\varepsilon^{3}\right),
\end{aligned}
$$

where $\hat{N}=Y^{\alpha \gamma} N_{\gamma \beta}$ and $N_{\alpha \beta}$ was defined in (5),

$$
\hat{H}=G^{i k}\left(\mathcal{D}_{k} \mathcal{D}_{j} S-\chi_{, k}^{\alpha} Y_{\alpha \beta} \chi_{, j}^{\beta}\right)
$$

takes into account the nontrivial geometry of the space of fields $\mathcal{M}$, and

$$
\begin{gathered}
\hat{U}_{1}=N^{\alpha \gamma} R_{\gamma}^{i}\left(\mathcal{D}_{i} R_{\delta}^{j}\right) \varepsilon_{j} N^{\delta \sigma} Y_{\sigma \beta}, \\
\hat{U}_{2}=N^{\alpha \gamma}\left(\mathcal{D}_{i} R_{\gamma}^{k}\right) \varepsilon_{k}\left(H^{-1}\right)^{i j}\left(\mathcal{D}_{j} R_{\delta}^{l}\right) \varepsilon_{l} N^{\delta \sigma} Y_{\sigma \beta}
\end{gathered}
$$

are two nonlocal operators responsible for restoring the offshell gauge independence of the one-loop effective action. In (17), $\hat{H}^{-1}$ is defined by the relation $\hat{H} \cdot \hat{H}^{-1}=-\hat{1}$ (of course, the latin indices $i, j, k \ldots$ should be raised and lowered with the metric $G^{i j}$ and its inverse). In the case of our interest, the terms of orders higher than $\varepsilon^{2}$ do not contribute to the divergent part of the one-loop effective action and therefore are not considered here.

It is worth noting that the latter feature is not true for other models of quantum gravity. In fact, in the higherderivative fourth-order gravity, only linear terms in $\varepsilon_{i}$ contribute to the divergences [31,32], while in quantum general relativity in higher dimensions, other terms are necessary. For explicit expressions of the $O\left(\varepsilon^{3}\right)$-terms, see Ref. [33]. Calculations of the unique effective action in $D \neq 4$ gravity models can be found, e.g., in Refs. [24, 33-37]. Even though we are mainly interested in $D=4$ 
results, for the sake of generality, we let the space-time dimension $D$ be arbitrary in our intermediate calculations.

\section{FIELD PARAMETRIZATIONS AND BILINEAR FORM OF THE ACTION}

In the traditional background field method, the original field $g_{\mu \nu}^{\prime}$ is split into a sum of a classical background $g_{\mu \nu}$ and a quantum field $h_{\mu \nu}$, i.e., $g_{\mu \nu}^{\prime}=g_{\mu \nu}+\kappa h_{\mu \nu}$. As in the present work we are interested in evaluating the one-loop divergences in a general parametrization of the quantum field, instead of performing the usual linear shift, we shall consider $g_{\mu \nu}^{\prime}=f_{\mu \nu}\left(g_{\alpha \beta}, \phi_{\alpha \beta}\right)$. Here, the indices are lowered and raised with the external metric $g_{\mu \nu}$ (and its inverse $g^{\mu \nu}$ ), and $f$ depends on the quantum field $\phi_{\mu \nu}$ possibly in a nonlinear way. Assuming that $f$ has a series expansion, we can define the most general (at one-loop order) parametrization of the quantum metric in the form [9]

$g_{\mu \nu}^{\prime}=g_{\mu \nu}+\kappa A_{(1) \mu \nu}^{\alpha \beta} \phi_{\alpha \beta}+\kappa^{2} A_{(2) \mu \nu}^{\lambda \tau, \rho \sigma} \phi_{\lambda \tau} \phi_{\rho \sigma}+O\left(\kappa^{3}\right)$,

where $A_{(n) \mu \nu}$ are tensor structures depending only on the background metric and $\kappa$ is the loop-expansion parameter. Through covariance and symmetry arguments, the coefficient functions in (18) have the general tensor form

$$
\begin{gathered}
A_{(1) \mu \nu}^{\alpha \beta}=\gamma_{1} \delta_{\mu \nu}^{\alpha \beta}+\gamma_{2} g^{\alpha \beta} g_{\mu \nu}, \\
A_{(2) \mu \nu}^{\lambda \tau, \rho \omega}=\frac{\gamma_{3}}{2} g^{\gamma \delta}\left(\delta_{\gamma(\mu}^{\lambda \tau} \delta_{\nu) \delta}^{\rho \omega}+\delta_{\gamma(\mu}^{\rho \omega} \delta_{\nu) \delta}^{\lambda \tau}\right)+\gamma_{4} \delta^{\lambda \tau, \rho \omega} g_{\mu \nu} \\
+\frac{\gamma_{5}}{2}\left(\delta_{\mu \nu}^{\lambda \tau} g^{\rho \omega}+\delta_{\mu \nu}^{\rho \omega} g^{\lambda \tau}\right)+\gamma_{6} g^{\lambda \tau} g^{\rho \omega} g_{\mu \nu} .
\end{gathered}
$$

In these expressions,

$$
\delta_{\alpha \beta}^{\mu \nu}=\frac{1}{2}\left(\delta_{\alpha}^{\mu} \delta_{\beta}^{\nu}+\delta_{\beta}^{\mu} \delta_{\alpha}^{\nu}\right)
$$

and $\gamma_{i}(i=1, \ldots, 6)$ are six arbitrary coefficients parametrizing the choice of the quantum variable. The restrictions $\gamma_{1} \neq 0$ and $\gamma_{1}+D \gamma_{2} \neq 0$ have to be imposed, to provide that the change of coordinates from $g_{\mu \nu}^{\prime}$ to $\phi_{\mu \nu}$ is not degenerate. Terms of order $O\left(\kappa^{3}\right)$ in (18) contribute only at the two- and higher-loop orders and hence are irrelevant and will be omitted in what follows. The one-loop contribution requires a functional integration of a quadratic form in $\phi_{\mu \nu}$, and hence it is evaluated taking $\kappa \rightarrow 0$ in Eq. (14).

Inserting expressions (19) and (20) in Eq. (18), we get

$$
\begin{aligned}
g_{\mu \nu}^{\prime}= & g_{\mu \nu}+\kappa\left(\gamma_{1} \phi_{\mu \nu}+\gamma_{2} \phi g_{\mu \nu}\right) \\
& +\kappa^{2}\left(\gamma_{3} \phi_{\mu \rho} \phi_{\nu}^{\rho}+\gamma_{4} g_{\mu \nu} \phi_{\rho \sigma} \phi^{\rho \sigma}\right. \\
& \left.+\gamma_{5} \phi \phi_{\mu \nu}+\gamma_{6} g_{\mu \nu} \phi^{2}\right)+O\left(\kappa^{3}\right),
\end{aligned}
$$

where $g^{\mu \nu} \phi_{\mu \nu} \equiv \phi$ denotes the trace of the quantum metric. Equation (22) represents a general parametrization of the
TABLE I. Values of the parameters in (22) for the covariant and contravariant densitized parametrizations.

\begin{tabular}{ccccccc}
\hline \hline & $\gamma_{1}$ & $\gamma_{2}$ & $\gamma_{3}$ & $\gamma_{4}$ & $\gamma_{5}$ & $\gamma_{6}$ \\
\hline$\left|g^{\prime}\right|^{p} g_{\mu \nu}^{\prime}$ & 1 & $p$ & 0 & $-p / 2$ & 0 & $p^{2} / 2$ \\
$\left|g^{\prime}\right|^{q} g^{\prime \mu \nu}$ & -1 & $-q$ & 1 & $q / 2$ & $q$ & $q^{2} / 2$ \\
\hline \hline
\end{tabular}

quantum metric for one-loop calculations. Other choices of quantum variables based on the expansions of $\left|g^{\prime}\right|^{p} g_{\mu \nu}^{\prime}$ and $\left|g^{\prime}\right|^{q} g^{\prime \mu \nu}$ (see, e.g., Refs. [7,8,38]) can be reduced to particular cases of (22). The explicit values of $\gamma_{i}$ for these parametrizations are displayed in Table I. Let us note that it is possible to construct a parametrization of the more general type $g_{\mu \nu}^{\prime}=e^{2 \kappa r \sigma}\left(g_{\mu \nu}+\cdots\right)$, in which the conformal factor $\sigma(x)$ of the metric is explicitly separated. Calculations using the conformal parametrization can be found, e.g., in Refs. $[6,8,9]$. We postpone the discussion on this choice to Sec. VI.

The bilinear form of the action can be obtained by expanding (1) in powers of $\phi_{\mu \nu}$ by means of (22). This yields [9]

$$
S\left(g_{\mu \nu}^{\prime}\right)=S\left(g_{\mu \nu}\right)+S^{(1)}+S^{(2)}+\cdots,
$$

where

$$
\begin{aligned}
S^{(1)}= & \frac{1}{\kappa} \int \mathrm{d}^{D} x \sqrt{|g|}\left\{\gamma_{1} R^{\mu \nu} \phi_{\mu \nu}\right. \\
& \left.-\frac{1}{2}\left[\gamma_{1}+(D-2) \gamma_{2}\right] R \phi-\left(\gamma_{1}+D\right) \gamma_{2} \Lambda \phi\right\},
\end{aligned}
$$

$$
\begin{aligned}
S^{(2)}= & -\frac{1}{2} \int \mathrm{d}^{D} x \sqrt{|g|}\left\{\phi _ { \mu \nu } \left[K^{\mu \nu, \alpha \beta}(\square-2 \Lambda)+M_{1}^{\mu \nu, \alpha \beta}\right.\right. \\
& \left.\left.+M_{2}^{\mu \nu, \alpha \beta}\right] \phi_{\alpha \beta}+\left(\gamma_{1} \nabla_{\rho} \phi_{\mu}^{\rho}+\beta \nabla_{\mu} \phi\right)^{2}\right\},
\end{aligned}
$$

and unnecessary superficial terms have been omitted. In the last formula,

$$
\beta=-\frac{1}{2}\left[\gamma_{1}+(D-2) \gamma_{2}\right],
$$

and the tensor objects are defined as

$$
\begin{aligned}
K^{\mu \nu, \alpha \beta}= & \frac{1}{2}\left\{\gamma_{1}^{2} \delta^{\mu \nu, \alpha \beta}-\frac{1}{2}\left[\gamma_{1}^{2}+2(D-2) \gamma_{1} \gamma_{2}\right.\right. \\
& \left.\left.+D(D-2) \gamma_{2}^{2}\right] g^{\mu \nu} g^{\alpha \beta}\right\}, \\
M_{1}^{\mu \nu, \alpha \beta}= & \gamma_{1}^{2} R^{\mu \alpha \nu \beta}+\gamma_{1}^{2} g^{\nu \beta} R^{\mu \alpha}-\frac{x_{1}}{2}\left(g^{\mu \nu} R^{\alpha \beta}+g^{\alpha \beta} R^{\mu \nu}\right) \\
& -\frac{\gamma_{1}^{2}}{2} \delta^{\mu \nu, \alpha \beta} R+\frac{x_{2}}{4} g^{\mu \nu} g^{\alpha \beta} R,
\end{aligned}
$$




$$
\begin{aligned}
M_{2}^{\mu \nu, \alpha \beta}= & -2 \gamma_{3} g^{\nu \beta} R^{\mu \alpha}-\gamma_{5}\left(g^{\mu \nu} R^{\alpha \beta}+g^{\alpha \beta} R^{\mu \nu}\right) \\
& +\left[\gamma_{3}+(D-2) \gamma_{4}\right] \delta^{\mu \nu, \alpha \beta} R \\
& +\left[\gamma_{5}+(D-2) \gamma_{6}\right] g^{\mu \nu} g^{\alpha \beta} R \\
& +2\left(\gamma_{3}+D \gamma_{4}\right) \delta^{\mu \nu, \alpha \beta} \Lambda+2\left(\gamma_{5}+D \gamma_{6}\right) g^{\mu \nu} g^{\alpha \beta} \Lambda,
\end{aligned}
$$

with

$$
\begin{aligned}
& x_{1}=\gamma_{1}^{2}+(D-4) \gamma_{1} \gamma_{2}, \\
& x_{2}=\gamma_{1}^{2}+2(D-4) \gamma_{1} \gamma_{2}+(D-2)(D-4) \gamma_{2}^{2} .
\end{aligned}
$$

It is worth noticing that all the dependences on the parameters $\gamma_{3, \ldots, 6}$ of the nonlinear part of the field splitting (22) are encoded in the tensor $M_{2}^{\mu \nu, \alpha \beta}$. In the above-given formulas, and in the following ones, we may present expressions in a compact form in which all algebraic symmetries are implicit (for more details, see Ref. [9]).

Finally, from Eq. (23), it follows that the equations of motion read

$$
\begin{aligned}
\varepsilon^{\mu \nu}= & \frac{1}{\sqrt{|g|}} \frac{\delta S}{\delta \phi_{\mu \nu}}=\frac{1}{\kappa}\left\{\gamma_{1} R^{\mu \nu}-\frac{1}{2}\left[\gamma_{1}+(D-2) \gamma_{2}\right] R g^{\mu \nu}\right. \\
& \left.-\left(\gamma_{1}+D\right) \gamma_{2} \Lambda g^{\mu \nu}+O(\kappa)\right\} .
\end{aligned}
$$

Now, we have all basic elements to perform the desired calculation.

\section{IMPROVED BILINEAR FORM OF THE ACTION}

General relativity and other metric theories of gravity are gauge theories based on the diffeomorphism group $\mathcal{G}$. The configuration space $\mathcal{M}$ is the set of all spacetime metrics, and the coset $\mathcal{M} / \mathcal{G}$ is known as the space of spacetime geometries. In quantum gravity, the invariant configurationspace metric is defined, up to an arbitrary real parameter $a$, by [39]

$$
\begin{aligned}
\delta s^{2} & =\int \mathrm{d}^{D} x \sqrt{\left|g^{\prime}\right|} G^{\prime \mu \nu, \alpha \beta} \delta g_{\mu \nu}^{\prime}(x) \delta g_{\alpha \beta}^{\prime}(x), \\
G^{\prime \mu \nu, \alpha \beta} & =\frac{1}{2}\left(\delta^{\prime \mu \nu, \alpha \beta}+a g^{\prime \mu \nu} g^{\prime \alpha \beta}\right) .
\end{aligned}
$$

The nondegeneracy of $G^{\prime \mu \nu, \alpha \beta}$ is ensured by the condition $a \neq-1 / D$. Explicit calculations have shown that the Vilkovisky effective action depends on the choice of $a$ $[15,24,40]$. The ambiguity owed to the parameter $a$ can be fixed by an additional prescription.

A differential operator is said to be minimal if its highestderivative term is given only by a power of the $\square$ operator. In quantum gravity models, the minimal operator almost always has the form of $G^{\mu \nu, \alpha \beta} \square^{n}$ with the parameter $a$ unambiguously fixed by the choice of classical Lagrangian and the parametrization of the quantum field. In Ref. [1], it was proposed that $a$ should be chosen correspondingly; namely, the field-space metric should be the expression in the highest-derivative term in the minimal version of the bilinear part of the classical action. This prescription relies on the assumption that all the geometrical objects underlying the framework of the unique effective action should be determined from the classical action [1]. For the quantum general relativity $n=1$ and in the standard simplest parametrization, this condition fixes the value $a=-1 / 2$. However, even in the minimal gauge, the coefficient of the term $g^{\mu \nu} g^{\alpha \beta}$ of the field-space metric may be changed by modifying the parametrization of the quantum metric, that is, by changing the coefficients $\gamma_{i}$ in (22) [see, for instance, Eq. (35) below]. One of the purposes of this work is to check whether this change produces a modification in the divergent part of the one-loop unique effective action.

The field-space metric in terms of the variable $\phi_{\mu \nu}$ can be obtained by performing a change of variables in Eq. (32), which gives

$$
\delta s^{2}=\int \mathrm{d}^{D} x \sqrt{|g|} G^{\mu \nu, \alpha \beta} \delta \phi_{\mu \nu}(x) \delta \phi_{\alpha \beta}(x),
$$

where

$$
\begin{gathered}
G^{\mu \nu, \alpha \beta}=G^{\mu \nu, \alpha \beta(0)}+\kappa G^{\mu \nu, \alpha \beta(1)}+O\left(\kappa^{2}\right), \\
G^{\mu \nu, \alpha \beta(0)}=\frac{1}{2}\left(\gamma_{1}^{2} \delta^{\mu \nu, \alpha \beta}+\bar{a} g^{\mu \nu} g^{\alpha \beta}\right), \\
\bar{a} \equiv \gamma_{2}\left(2 \gamma_{1}+D \gamma_{2}\right)+a\left(\gamma_{1}+D \gamma_{2}\right)^{2}, \\
G^{\mu \nu, \alpha \beta(1)}=g_{1} g^{\mu \alpha} \phi^{\nu \beta}+g_{2} \delta^{\mu \nu, \alpha \beta} \phi \\
+ \\
+g_{3}\left(g^{\mu \nu} \phi^{\alpha \beta}+g^{\alpha \beta} \phi^{\mu \nu}\right)+g_{4} g^{\mu \nu} g^{\alpha \beta} \phi,
\end{gathered}
$$

with the coefficients

$$
\begin{aligned}
g_{1}= & -\gamma_{1}^{3}+2 \gamma_{1} \gamma_{3}, \quad g_{2}=\frac{\gamma_{1}^{2}}{4}\left[\gamma_{1}+(D-4) \gamma_{2}\right]+\gamma_{1} \gamma_{5}, \\
g_{3}= & -\frac{\gamma_{1}^{2}}{2}\left[2 \gamma_{2}+a\left(\gamma_{1}+D \gamma_{2}\right)\right]+\gamma_{2} \gamma_{3} \\
& +\left(\gamma_{1}+D \gamma_{2}\right)\left[\gamma_{4}+a\left(\gamma_{3}+D \gamma_{4}\right)\right]+\frac{\gamma_{1} \gamma_{5}}{2}, \\
g_{4}= & \frac{\bar{a}}{4}\left[\gamma_{1}+(D-4) \gamma_{2}\right]-\gamma_{1} \gamma_{2}\left[\gamma_{2}+a\left(\gamma_{1}+D \gamma_{2}\right)\right] \\
& +2\left[\gamma_{1} \gamma_{6}+\gamma_{2}\left(\gamma_{5}+D \gamma_{6}\right)+a\left(\gamma_{1}+D \gamma_{2}\right)\left(\gamma_{5}+D \gamma_{6}\right)\right] .
\end{aligned}
$$

Formula (35) can be rewritten using the definition of Eq. (27), 
$G^{\mu \nu, \alpha \beta(0)}=K^{\mu \nu, \alpha \beta}+\frac{1}{4}(1+2 a)\left(\gamma_{1}+D \gamma_{2}\right)^{2} g^{\mu \nu} g^{\alpha \beta}$.

One can see that for $a=-1 / 2$ the background configuration space metric reduces to the factor of the d'Alembertian in Eq. (25). This agrees with the Vilkovisky's prescription [1] for fixing the ambiguity in the one-parameter family of metrics, even for the general parametrization (22).

The Christoffel symbol (8) associated with the metric (34) has the form

$$
\Gamma_{\rho \sigma}^{\mu \nu, \alpha \beta}=\frac{1}{2} G_{\rho \sigma, \lambda \tau}\left(\frac{\partial G^{\lambda \tau, \alpha \beta}}{\partial \phi_{\mu \nu}}+\frac{\partial G^{\mu \nu, \lambda \tau}}{\partial \phi_{\alpha \beta}}-\frac{\partial G^{\mu \nu, \alpha \beta}}{\partial \phi_{\lambda \tau}}\right),
$$

where the inverse of the configuration-space metric (34) is

$$
\begin{aligned}
G_{\mu \nu, \alpha \beta}= & K_{\mu \nu, \alpha \beta}^{-1}+\frac{2(1+2 a)}{(D-2)(1+a D)\left(\gamma_{1}+D \gamma_{2}\right)^{2}} g_{\mu \nu} g_{\alpha \beta} \\
& +O(\kappa)
\end{aligned}
$$

and $K_{\mu \nu, \alpha \beta}^{-1}$ is the inverse of (27),

$$
K_{\mu \nu, \alpha \beta}^{-1}=h_{1} \delta_{\mu \nu, \alpha \beta}+h_{2} g_{\mu \nu} g_{\alpha \beta},
$$

with

$$
h_{1}=\frac{2}{\gamma_{1}^{2}}, \quad h_{2}=-\frac{2}{D \gamma_{1}^{2}}-\frac{4}{D(D-2)\left(\gamma_{1}+D \gamma_{2}\right)^{2}} .
$$

A straightforward calculation of (39) yields

$$
\begin{aligned}
\Gamma_{\rho \sigma}^{\mu \nu, \alpha \beta}= & \kappa\left[c_{1} \delta_{\rho \sigma}^{\mu \alpha} g^{\nu \beta}+c_{2}\left(\delta_{\rho \sigma}^{\mu \nu} g^{\alpha \beta}+\delta_{\rho \sigma}^{\alpha \beta} g^{\mu \nu}\right)\right. \\
& \left.+c_{3} \delta^{\mu \nu, \alpha \beta} g_{\rho \sigma}+c_{4} g^{\mu \nu} g^{\alpha \beta} g_{\rho \sigma}\right]+O\left(\kappa^{2}\right),
\end{aligned}
$$

where the coefficients are

$$
\begin{aligned}
& c_{1}=-\gamma_{1}+2 \frac{\gamma_{3}}{\gamma_{1}}, \quad c_{2}=\frac{1}{4}\left[\gamma_{1}+(D-4) \gamma_{2}\right]+\frac{\gamma_{5}}{\gamma_{1}}, \\
& c_{3}=\frac{1}{2(D-2)\left(\gamma_{1}+D \gamma_{2}\right)}\left[\gamma_{1}^{2}+2(D-2) \gamma_{1} \gamma_{2}-\frac{(1+2 a) D \gamma_{1}^{2}}{2(1+a D)}\right]+2 \frac{\gamma_{1} \gamma_{4}-\gamma_{2} \gamma_{3}}{\gamma_{1}\left(\gamma_{1}+D \gamma_{2}\right)}, \\
& c_{4}=-\frac{1}{4(D-2)\left(\gamma_{1}+D \gamma_{2}\right)}\left[\gamma_{1}^{2}+2(D-4) \gamma_{1} \gamma_{2}+(D-2)(D-4) \gamma_{2}^{2}-\frac{(1+2 a) \gamma_{1}^{2}}{(1+a D)}\right]+2 \frac{\gamma_{1} \gamma_{6}-\gamma_{2} \gamma_{5}}{\gamma_{1}\left(\gamma_{1}+D \gamma_{2}\right)} .
\end{aligned}
$$

Using Eqs. (31) and (43), the Christoffel correction term in the second covariant derivative $\mathcal{D}_{i} \mathcal{D}_{j} S=S_{, i j}-\Gamma_{i j}^{k} \varepsilon_{k}$ reads

$$
\begin{aligned}
\left.\Gamma_{\rho \sigma}^{\mu \nu, \alpha \beta} \varepsilon^{\rho \sigma}\right|_{\kappa \rightarrow 0}= & \frac{x_{1}}{4}\left(g^{\mu \nu} R^{\alpha \beta}+g^{\alpha \beta} R^{\mu \nu}\right)-\gamma_{1}^{2} g^{\mu \alpha} R^{\nu \beta}+\frac{\gamma_{1}^{2}}{4} \delta^{\mu \nu, \alpha \beta} R-\frac{x_{2}}{8} g^{\mu \nu} g^{\alpha \beta} R \\
& -M_{2}^{\mu \nu, \alpha \beta}+\frac{D-4}{D-2} K^{\mu \nu, \alpha \beta} \Lambda+\frac{(1+2 a) D \gamma_{1}^{2}}{8(1+a D)}\left(R+\frac{2 D}{D-2} \Lambda\right)\left(\delta^{\mu \nu, \alpha \beta}-\frac{1}{D} g^{\alpha \beta} g^{\mu \nu}\right),
\end{aligned}
$$

where $M_{2}^{\mu \nu, \alpha \beta}$ and $x_{1,2}$ were defined in Eqs. (29) and (30), respectively. We remark that the parameters $\gamma_{3, \ldots, 6}$, which are related to the nonlinear terms in the parametrization (22), only occur in $M_{2}^{\mu \nu, \alpha \beta}$, just as in (25). Because of this, the second functional covariant derivative of the action (23) only depends on the parameters $\gamma_{1}$ and $\gamma_{2}$,

$$
\begin{aligned}
-\left.\frac{\mathcal{D}^{2} S}{\delta \phi_{\mu \nu} \delta \phi_{\alpha \beta}}\right|_{\kappa \rightarrow 0}= & \frac{\gamma_{1}^{2}}{2} \delta^{\mu \nu, \alpha \beta} \square-\frac{d_{1}}{2} g^{\mu \nu} g^{\alpha \beta} \square+\frac{d_{2}}{2}\left(g^{\mu \nu} \nabla^{\alpha} \nabla^{\beta}+g^{\alpha \beta} \nabla^{\mu} \nabla^{\nu}\right) \\
& -\gamma_{1}^{2} g^{\mu \alpha} \nabla^{\nu \beta}+\gamma_{1}^{2} R^{\mu \alpha \nu \beta}-\frac{x_{1}}{4}\left(g^{\mu \nu} R^{\alpha \beta}+g^{\alpha \beta} R^{\mu \nu}\right)-\frac{\gamma_{1}^{2}}{4} \delta^{\mu \nu, \alpha \beta} R+\frac{x_{2}}{8} g^{\mu \nu} g^{\alpha \beta} R \\
& -\frac{D}{D-2} K^{\mu \nu, \alpha \beta} \Lambda+\frac{(1+2 a) D \gamma_{1}^{2}}{8(1+a D)}\left(\delta^{\mu \nu, \alpha \beta}-\frac{1}{D} g^{\alpha \beta} g^{\mu \nu}\right)\left(R+\frac{2 D}{D-2} \Lambda\right),
\end{aligned}
$$

where

$$
d_{1}=\gamma_{1}^{2}+2(D-2) \gamma_{1} \gamma_{2}+(D-1)(D-2) \gamma_{2}^{2}, \quad d_{2}=\gamma_{1}^{2}+(D-2) \gamma_{1} \gamma_{2} \text {. }
$$


It is clear that the Christoffel symbol derived from the metric (34) should suffice to compensate the dependence of $S_{, i j}$ on the nonlinearity of the field parametrization. In fact, for $\kappa \rightarrow 0$, all the parameters $\gamma_{3, \ldots, 6}$ only contribute to the last term in the rhs of

$$
\frac{\delta^{2} S^{\prime}}{\delta g_{\mu \nu}^{\prime} \delta g_{\alpha \beta}^{\prime}}=\frac{\delta \phi_{\lambda \tau}}{\delta g_{\mu \nu}^{\prime}} \frac{\delta \phi_{\rho \sigma}}{\delta g_{\alpha \beta}^{\prime}} \frac{\delta^{2} S}{\delta \phi_{\lambda \tau} \delta \phi_{\rho \sigma}}+\frac{\delta^{2} \phi_{\lambda \tau}}{\delta g_{\mu \nu}^{\prime} \delta g_{\alpha \beta}^{\prime}} \frac{\delta S}{\delta \phi_{\lambda \tau}}
$$

which represents the nontensor nature of this transformation.

\section{ONE-LOOP DIVERGENCES OF VILKOVISKY EFFECTIVE ACTION}

Up to this point, we have considered the part of the Vilkovisky effective action based on the Christoffel symbols on the space $\mathcal{M}$ of field parametrization. However, it is still necessary to introduce the gauge fixing for the diffeomorphism invariance and take into account the contribution of the Faddeev-Popov ghosts as well the terms (16) and (17) related to the gauge constraints on the affine connection.

The standard general form of the gauge-fixing action in quantum general relativity is

$$
S_{\mathrm{GF}}=\frac{1}{2} \int \mathrm{d}^{D} x \sqrt{|g|} \chi_{\alpha} g^{\alpha \beta} \chi_{\beta}
$$

where $\chi_{\alpha}$ is the background gauge condition. The use of a linear gauge fixing ${ }^{2}$ is not a necessary condition to ensure the invariance of the Vilkovisky effective action [22,27]. Nonetheless, as explained in Sec. II, the DeWitt gauge (13) is crucial for deriving the expanded formula (14). In our parametrization, it assumes the form

$$
\begin{aligned}
\chi_{\alpha} & =G^{\mu \nu, \lambda \tau} R_{\mu \nu, \alpha} \phi_{\lambda \tau} \\
& =-\gamma_{1} \nabla_{\rho} \phi_{\alpha}^{\rho}-\left[\gamma_{2}+a\left(\gamma_{1}+D \gamma_{2}\right)\right] \nabla_{\alpha} \phi+O(\kappa),
\end{aligned}
$$

where we used the explicit expression for the generators of the gauge transformations $R_{\mu \nu, \alpha}$ of the field $\phi_{\mu \nu}$, presented in the Appendix.

Comparing Eqs. (49) and (25), it is easy to see that the choice $a=-1 / 2$ provides the minimal form of the operator (15),

$$
\hat{H}=\left.G_{\mu \nu, \rho \sigma}\left(\frac{\mathcal{D}^{2} S}{\delta \phi_{\rho \sigma} \delta \phi_{\alpha \beta}}+\frac{\delta \chi_{\lambda}}{\delta \phi_{\rho \sigma}} g^{\lambda \tau} \frac{\delta \chi_{\tau}}{\delta \phi_{\alpha \beta}}\right)\right|_{\kappa \rightarrow 0} .
$$

\footnotetext{
${ }^{2}$ See Ref. [41] for a recent discussion on nonlinear gauges within the framework of the background field method in the standard definition of the effective action.
}

Let us remark that another possible way of making the operator $H^{\mu \nu, \alpha \beta}$ minimal is through the use of a specific parametrization, namely, $\gamma_{1}=-D \gamma_{2}$. However, as explained in Sec. III, this is not acceptable since it makes the metric in the space of the quantum fields singular, see Eq. (40), and the operator $\hat{H}$ in (50) undefined. Thus, $a=-1 / 2$ is the sole reasonable choice. For this value of $a$, the operator gets reduced to the standard form

$$
\hat{H}=-(\hat{1} \square+\hat{\Pi}),
$$

where $\hat{1}=\delta_{\alpha \beta}^{\mu \nu}$ is the identity operator (21) on the space of symmetric rank- 2 tensors and

$$
\begin{aligned}
\hat{\Pi}= & 2 R_{. \alpha . \beta}^{\mu \nu}-\frac{p_{1}}{2} g^{\mu \nu} R_{\alpha \beta}-\frac{p_{2}}{D-2} g_{\alpha \beta} R^{\mu \nu} \\
& +\frac{p_{3}}{2(D-2)} g^{\mu \nu} g_{\alpha \beta} R+\delta_{\alpha \beta}^{\mu \nu}\left(\frac{D \Lambda}{D-2}-\frac{1}{2} R\right),
\end{aligned}
$$

with

$$
\begin{aligned}
& p_{1}=1+\frac{\gamma_{2}(D-4)}{\gamma_{1}}, \\
& p_{2}=\frac{\gamma_{1}+2(D-2) \gamma_{2}}{\gamma_{1}+D \gamma_{2}}, \\
& p_{3}=p_{2}+\frac{(D-2)(D-4) \gamma_{2}^{2}}{\gamma_{1}\left(\gamma_{1}+D \gamma_{2}\right)} .
\end{aligned}
$$

Furthermore, with the gauge condition (49), the ghost matrix reads

$$
\begin{aligned}
\hat{N} & =g^{\alpha \lambda} G^{\mu \nu, \rho \sigma} R_{\mu \nu, \lambda} R_{\rho \sigma, \beta} \\
& =\delta_{\beta}^{\alpha} \square+(1+2 a) \nabla^{\alpha} \nabla_{\beta}+R_{\beta}^{\alpha}+O(\kappa) .
\end{aligned}
$$

Notice that in the DeWitt gauge all the dependence on the parametrization is canceled in the ghost operator and that $a=-1 / 2$ makes it also minimal. Hereafter, we choose this value for $a$, such that both $\hat{H}$ and $\hat{N}$ assume minimal forms.

The correction which is responsible to restore the gauge invariance of the effective action is based on the nonlocal operators $\hat{U}_{1}$ and $\hat{U}_{2}$, defined in (16) and (17). These operators depend on the two new vertices

$$
\left(V_{1}\right)_{i \alpha}=\left(\mathcal{D}_{i} R_{\alpha}^{j}\right) \varepsilon_{j} \quad \text { and } \quad\left(V_{2}\right)_{\alpha \beta}=R_{\alpha}^{i}\left(\mathcal{D}_{i} R_{\beta}^{j}\right) \varepsilon_{j} .
$$

Particularizing the formulas above for the gravity theory in the parametrization (22) and using the gauge generators (A4) given in Appendix, after some algebra, we get 


$$
\begin{aligned}
\left(V_{1}\right)_{\gamma}^{\mu \nu}= & \frac{\gamma_{1}}{2}\left(R_{\gamma}^{\mu} \nabla^{\nu}+R_{\gamma}^{\nu} \nabla^{\mu}\right)-\frac{\gamma_{1}}{2}\left(\delta_{\gamma}^{\mu} R^{\nu \lambda}+\delta_{\gamma}^{\nu} R^{\mu \lambda}\right) \nabla_{\lambda}+\gamma_{1}\left(\nabla_{\gamma} R^{\mu \nu}\right) \\
& +\frac{\gamma_{1}}{2} R^{\mu \nu} \nabla_{\gamma}-\frac{1}{2}\left(\gamma_{1}+D \gamma_{2}\right) g^{\mu \nu} R_{\gamma}^{\lambda} \nabla_{\lambda}+\frac{\gamma_{1}}{4} R\left(\delta_{\gamma}^{\mu} \nabla^{\nu}+\delta_{\gamma}^{\nu} \nabla^{\mu}\right) \\
& -\frac{1}{2}\left[\gamma_{1}+(D-2) \gamma_{2}\right] g^{\mu \nu}\left(\nabla_{\gamma} R\right)-\frac{1}{4}\left[\gamma_{1}+(D-4) \gamma_{2}\right] g^{\mu \nu} R \nabla_{\gamma} \\
& +\frac{D \gamma_{1}}{2(D-2)} \Lambda\left(\delta_{\gamma}^{\mu} \nabla^{\nu}+\delta_{\gamma}^{\nu} \nabla^{\mu}\right)-\frac{D\left[\gamma_{1}+(D-2) \gamma_{2}\right]}{2(D-2)} g^{\mu \nu} \Lambda \nabla_{\gamma}+O(\kappa)
\end{aligned}
$$

and

$$
\begin{aligned}
\left(V_{2}\right)_{\alpha \beta}= & R_{\alpha \beta} \square+\frac{1}{2} g_{\alpha \beta} R \square-g_{\alpha \beta} R^{\lambda \tau} \nabla_{\lambda} \nabla_{\tau} \\
& +\left(\nabla^{\lambda} R_{\alpha \beta}\right) \nabla_{\lambda}-\left(\nabla_{\alpha} R_{\beta}^{\lambda}\right) \nabla_{\lambda}+\left(\nabla_{\beta} R_{\alpha}^{\lambda}\right) \nabla_{\lambda} \\
& -R_{\alpha \lambda \beta \tau} R^{\lambda \tau}+R_{\alpha \lambda} R_{\beta}^{\lambda}+\frac{1}{2} R R_{\alpha \beta} \\
& +\frac{D \Lambda}{D-2}\left(g_{\alpha \beta} \square+R_{\alpha \beta}\right)+O(\kappa) .
\end{aligned}
$$

We see that the dependence on the parameters $\gamma_{3, \ldots, 6}$ corresponding to the nonlinear part of the field splitting (22) gets canceled in $\left(V_{1}\right)_{\gamma}^{\mu \nu}$, while the vertex $\left(V_{2}\right)_{\alpha \beta}$ is parametrization independent automatically.

The operators $\hat{U}_{1}$ and $\hat{U}_{2}$ can be obtained by substituting the two previous equations into the formulas (16) and (17), together with the propagators

$$
\begin{aligned}
N^{\alpha \beta} & =-g^{\alpha \beta} \frac{1}{\square}+R^{\alpha \beta} \frac{1}{\square^{2}}+O\left([m]^{3}\right), \\
H_{\mu \nu, \alpha \beta}^{-1} & =K_{\mu \nu, \alpha \beta}^{-1} \frac{1}{\square}+O\left([m]^{2}\right) .
\end{aligned}
$$

Here, $O\left([m]^{k}\right)$ denotes a series of inessential terms of higher background dimension $k$. Remember that, according to Ref. [17], for a functional universal trace

$$
\operatorname{Tr} \hat{C}^{\mu_{1} \cdots \mu_{k}} \nabla_{\mu_{1}} \cdots \nabla_{\mu_{k}} \frac{\hat{1}}{\square^{n}},
$$

the background dimension (in mass units) is defined as the dimension of the tensorial coefficient $\hat{C}^{\mu_{1} \cdots \mu_{k}}$, and its superficial degree of divergence is expressed by the relation $\omega=D-2 n+k$. Thus, in four dimensions, only the traces with background dimension $0,1,2,3$, and 4 contribute to the UV divergences.

With all these ingredients in hand, it is possible to evaluate the contribution of each term in (14), up to background dimension $O\left([\mathrm{~m}]^{4}\right)$, to the effective action. In the case of the operators $\hat{H}$ and $\hat{N}$ [respectively given by Eqs. (51) and (53)], this can be obtained from the functional trace of the coefficient $\hat{a}_{2}$ of the Schwinger-DeWitt expansion [16]. On the other hand, the functional traces of the nonlocal operators $\hat{U}_{1}, \hat{U}_{1}^{2}$, and $\hat{U}_{2}$ can be evaluated using the table of universal functional traces within the generalized Schwinger-DeWitt technique [17]. For example, one can easily show that

$\operatorname{Tr} \hat{U}_{2}=\left.\int \mathrm{d}^{D} x \operatorname{tr}\left[h_{1}\left(V_{1}^{2}\right)_{\beta}^{\alpha}+h_{2}\left(\bar{V}_{1}^{2}\right)_{\beta}^{\alpha}\right] \frac{1}{\square^{3}}\right|_{x^{\prime} \rightarrow x}+O\left([m]^{5}\right)$,

where $h_{1,2}$ were defined in Eq. (42) and we used the notations

$$
\begin{aligned}
& \left(V_{1}^{2}\right)_{\beta}^{\alpha}=g^{\alpha \gamma} \delta_{\mu \nu, \rho \sigma}\left(V_{1}\right)_{\gamma}^{\mu \nu}\left(V_{1}\right)_{\beta}^{\rho \sigma}, \quad\left(\bar{V}_{1}\right)_{\gamma}=g_{\mu \nu}\left(V_{1}\right)_{\gamma}^{\mu \nu}, \\
& \left(\bar{V}_{1}^{2}\right)_{\beta}^{\alpha}=g^{\alpha \gamma}\left(\bar{V}_{1}\right)_{\gamma}\left(\bar{V}_{1}\right)_{\beta} .
\end{aligned}
$$

Skipping the algebra, the contributions of the terms in (14) to the $\frac{1}{D-4}$-pole of the Vilkovisky unique effective action is presented in Table II. It is important to recall that only in $D \rightarrow 4$ the displayed coefficients correspond to one-loop divergences; nonetheless, our calculation in arbitrary dimension shows that they do not depend on the field parametrization even for $D \neq 4$. Moreover, one can see that the parametrization dependence which remained after the Christoffel correction was taken into account is canceled in the functional trace of each operator on its turn, as none of the coefficients depends on $\gamma_{1,2}$.

Since the object of our interest is the one-loop logarithmically divergent part of the Vilkovisky effective action, in the framework of dimensional regularization, we can take the limit $D \rightarrow 4$ in the coefficient of the pole term, to obtain

$$
\begin{aligned}
\bar{\Gamma}_{\mathrm{div}}^{(1)}= & -\frac{\mu^{D-4}}{(4 \pi)^{2}(D-4)} \int \mathrm{d}^{D} x \sqrt{|g|} \\
& \times\left\{\frac{53}{45} R_{\mu \nu \alpha \beta}^{2}-\frac{61}{90} R_{\mu \nu}^{2}+\frac{25}{36} R^{2}+8 \Lambda R+12 \Lambda^{2}\right\} .
\end{aligned}
$$

As usual, $\mu$ is the renormalization parameter. Formula (60) reproduces the results for the Vilkovisky effective action for general relativity calculated in the standard, particular, parametrization of the quantum variables in Ref. [17] (the coefficients of the terms related to the cosmological constant were calculated for the first time in Ref. [22]). Moreover, it is straightforward to verify that, on the classical mass shell, the divergences of Eq. (60) correctly 
TABLE II. Contribution of each operator in (14) to the coefficients of each curvature invariant in the divergent (at $D \rightarrow 4$ ) part of the one-loop Vilkovisky effective action. Each invariant enters the effective action multiplied by the overall coefficient as in Eq. (60). The final coefficients, which are the sum of the coefficients of columns 2-6, are presented in the last column.

\begin{tabular}{lcccccc}
\hline \hline Invariant & $\frac{i}{2} \operatorname{Tr} \ln \hat{H}$ & $-i \operatorname{Tr} \ln \hat{N}$ & $-\frac{i}{2} \operatorname{Tr} \hat{U}_{1}$ & $-\frac{i}{4} \operatorname{Tr} \hat{U}_{1}^{2}$ & $\frac{i}{2} \operatorname{Tr} \hat{U}_{2}$ & $\bar{\Gamma}^{(1)}$ \\
\hline$R_{\mu \nu \alpha \beta}^{2}$ & $\frac{D^{2}-29 D+480}{360}$ & $\frac{15-D}{90}$ & 0 & 0 & 0 & $\frac{D^{2}-33 D+540}{360}$ \\
$R_{\mu \nu}^{2}$ & $-\frac{D\left(D^{2}-D+178\right)}{360(D-2)}$ & $\frac{D-90}{90}$ & $\frac{D+12}{6}$ & $\frac{D+12}{24}$ & $-\frac{3 D^{2}-16}{8(D-2)}$ & $-\frac{D^{3}+55 D^{2}-204 D+360}{360(D-2)}$ \\
$R^{2}$ & $\frac{D^{3}-D^{2}+10 D-6}{36(D-2)}$ & $-\frac{D+12}{36}$ & $\frac{1}{6}$ & $\frac{D+12}{48}$ & $-\frac{3 D-4}{8(D-2)}$ & $\frac{4 D^{3}-5 D^{2}+24}{144(D-2)}$ \\
$\Lambda R$ & $\frac{D\left(D^{2}+D+6\right)}{6(D-2)}$ & 0 & $\frac{D(D+6)}{6(D-2)}$ & $\frac{D(D+4)}{4(D-2)}$ & $-\frac{D(D+4)}{2(D-2)}$ & $\frac{D\left(2 D^{2}+D+12\right)}{12(D-2)}$ \\
$\Lambda^{2}$ & $\frac{D^{3}(D+1)}{4(D-2)^{2}}$ & 0 & 0 & $\frac{D^{3}}{2(D-2)^{2}}$ & $-\frac{D^{3}}{(D-2)^{2}}$ & $\frac{D^{3}(D-1)}{4(D-2)^{2}}$ \\
\hline \hline
\end{tabular}

reduce to the coefficients of the usual on-shell effective action $[3,42]$,

$$
\begin{aligned}
\left.\bar{\Gamma}_{\text {div }}^{(1)}\right|_{\text {on-shell }}= & -\frac{\mu^{D-4}}{(4 \pi)^{2}(D-4)} \int \mathrm{d}^{D} x \sqrt{|g|} \\
& \times\left\{\frac{53}{45} R_{\mu \nu \alpha \beta}^{2}-\frac{58}{5} \Lambda^{2}\right\} .
\end{aligned}
$$

This is an expected result since the Vilkovisky correction term is proportional to the equations of motion. On the other hand, this result is known to be gauge-fixing and parametrization independent [9].

It is interesting to compare the result for the unique effective action (60) and the one-loop divergences of the standard (usual) effective action in an arbitrary parametrization (22), derived in Ref. [9]. It turns out that the two expressions coincide if the parameters satisfy the conditions

$$
\begin{aligned}
& \gamma_{4}=\frac{1}{48}\left[(6 \pm \sqrt{15}) \gamma_{1}^{2}-12 \gamma_{3}\right], \\
& \gamma_{5}=\frac{1}{12}\left[-6 \gamma_{3} \pm\left(1+\frac{4 \gamma_{2}}{\gamma_{1}}\right) \sqrt{6\left(12 \gamma_{3}^{2}-5 \gamma_{1}^{4}\right)}\right], \\
& \gamma_{6}=-\frac{1}{64}\left[5\left(\gamma_{1}+4 \gamma_{2}\right)^{2}+4\left[\gamma_{3}+4\left(\gamma_{4}+\gamma_{5}\right)\right]\right] .
\end{aligned}
$$

In this case, the one-loop divergences of the conventional effective action calculated in the minimal gauge coincide with those of the Vilkovisky effective action (60). Curiously, this result can be achieved only if the parametrization is nonlinear. This can be readily seen from Eq. (63), which implies $\gamma_{3} \neq 0$. Let us note that the observation formulated above can be seen as a parametrization-dependence counterpart for the result of Ref. [43], where it was derived a gauge for which the one-loop divergences of the conventional effective action (in the particular simplest parametrization) reproduce those of the unique effective action. In this vein, it is also worth pointing out that the $\Lambda$-dependent terms in (60) can be obtained by means of the Landau-DeWitt gauge within the usual definition of the effective action [22]. Nevertheless, the simple use of this particular singular gauge in the standard effective action cannot give the other divergent terms of the unique effective action for Einstein gravity because the space of fields is not flat [22,27,29].

\section{CONFORMAL PARAMETRIZATION OF THE METRIC}

Let us now consider a more general parametrization of the metric, which explicitly splits its conformal factor, namely,

$$
\begin{aligned}
g_{\mu \nu}^{\prime}= & e^{2 \kappa r \sigma}\left[g_{\mu \nu}+\kappa\left(\gamma_{1} \phi_{\mu \nu}+\gamma_{2} \phi g_{\mu \nu}\right)\right. \\
& +\kappa^{2}\left(\gamma_{3} \phi_{\mu \rho} \phi_{\nu}^{\rho}+\gamma_{4} g_{\mu \nu} \phi_{\rho \sigma}^{2}+\gamma_{5} \phi \phi_{\mu \nu}+\gamma_{6} \phi^{2} g_{\mu \nu}\right) \\
& \left.+O\left(\kappa^{3}\right)\right]
\end{aligned}
$$

where $g_{\mu \nu}$ is the background metric, $\phi_{\mu \nu}$ and $\sigma$ are the quantum fields, and $\gamma_{1, \ldots, 6}$ and $r$ are arbitrary parameters. The one-loop divergences of the standard effective action for Einstein gravity were evaluated in this parametrization in Ref. [9].

It turns out that it is not possible to construct the Vilkovisky effective action directly in this parametrization. Treating the conformal factor $\sigma$ as a new field increases the total number of scalar modes. As a consequence, there is an artificial conformal symmetry and related degeneracy, making the transformation singular. For instance, the metric in the configuration space is

$$
G^{A B}=\left(\begin{array}{cc}
G^{\mu \nu, \alpha \beta(0)} & r\left(\gamma_{1}+D \gamma_{2}\right)(1+a D) g^{\mu \nu} \\
r\left(\gamma_{1}+D \gamma_{2}\right)(1+a D) g^{\alpha \beta} & 2 r^{2} D(1+a D)
\end{array}\right)+O(\kappa)
$$


where $A, B, \cdots$ take the labels $\phi_{\mu \nu}, \sigma$, and $G^{\mu \nu, \alpha \beta(0)}$ coincides with Eq. (38). The determinant of the $O\left(\kappa^{0}\right)$ term of this metric reads

$$
\begin{aligned}
\left|G^{A B(0)}\right|= & \left\{2 r^{2} D(1+a D)-r^{2}\left(\gamma_{1}+D \gamma_{2}\right)^{2}\right. \\
& \left.\times(1+a D)^{2} g^{\mu \nu} g^{\alpha \beta} G_{\mu \nu, \alpha \beta}^{(0)}\right\} \times\left|G^{\mu \nu, \alpha \beta(0)}\right| .
\end{aligned}
$$

It is straightforward to verify that the term in curly brackets vanishes, proving that the field-space metric is degenerate. Therefore, it is not possible to evaluate the Christoffel symbols. The problem originates from the fact that the change of variables $g_{\mu \nu}^{\prime} \mapsto\left(\phi_{\mu \nu}, \sigma\right)$ is not a diffeomorphism.

The described difficulty can be resolved as follows. We impose, from the beginning, the additional conformal gauge fixing

$$
\sigma=\lambda \phi
$$

with $\lambda$ being the gauge-fixing parameter. Expanding the exponential in (65), one can see that, up to order $\kappa^{2}$, this parametrization reduces to (22) via the substitutions

$\gamma_{2} \mapsto \gamma_{2}+2 r \lambda, \quad \gamma_{5} \mapsto \gamma_{5}+2 r \lambda \gamma_{1}, \quad \gamma_{6} \mapsto \gamma_{6}+2 r \lambda \gamma_{1}$.

Then, all calculations that we carried out for (22) also apply for the conformal parametrization (65).

An alternative approach is to split the field $\phi_{\mu \nu}$ in the trace and traceless part, that is,

$$
\phi_{\mu \nu}=\bar{\phi}_{\mu \nu}+\frac{1}{D} g_{\mu \nu} \phi
$$

It is clear that $g^{\mu \nu} \bar{\phi}_{\mu \nu}=0$. We now have a parametrization in terms of two independent quantum fields: $\bar{\phi}_{\mu \nu}$ and $\phi$. Applying (68) and (70) in (65), we get

$$
\begin{aligned}
g_{\alpha \beta}^{\prime}= & g_{\alpha \beta}+\kappa\left(\gamma_{1} \bar{\phi}_{\alpha \beta}+\bar{\gamma}_{2} \phi g_{\alpha \beta}\right) \\
& +\kappa^{2}\left(\gamma_{3} \bar{\phi}_{\alpha \rho} \bar{\phi}_{\beta}^{\rho}+\gamma_{4} \bar{\phi}_{\rho \sigma} \bar{\phi}^{\rho \sigma} g_{\alpha \beta}\right. \\
& \left.+\bar{\gamma}_{5} \phi \bar{\phi}_{\alpha \beta}+\bar{\gamma}_{6} \phi^{2} g_{\alpha \beta}\right)+O\left(\kappa^{3}\right),
\end{aligned}
$$

where the new coefficients are

$$
\begin{aligned}
\bar{\gamma}_{2}= & \frac{\gamma_{1}}{D}+\gamma_{2}+2 r \lambda, \\
\bar{\gamma}_{5}= & \frac{2 \gamma_{3}}{D}+\gamma_{5}+2 \gamma_{1} r \lambda, \\
\bar{\gamma}_{6}= & \frac{1}{D^{2}}\left[\gamma_{3}+D\left(\gamma_{4}+\gamma_{5}\right)+D^{2} \gamma_{6}\right. \\
& \left.+2 D\left(\gamma_{1}+D \gamma_{2}\right) r \lambda\right]+2 r^{2} \lambda^{2} .
\end{aligned}
$$

Now, it is possible to define a nonsingular metric in the space of the fields, ${ }^{3}$

$$
\begin{aligned}
G^{\bar{\phi}_{\mu \nu}, \bar{\phi}_{\alpha \beta}} & =\gamma_{1}^{2} \bar{\delta}^{\mu \nu, \alpha \beta}+\kappa\left[\zeta_{1} g^{\mu \alpha} \bar{\phi}^{\beta \nu}+\zeta_{2} \bar{\delta}^{\mu \nu, \alpha \beta} \phi\right]+O\left(\kappa^{2}\right), \\
G^{\bar{\phi}_{\alpha \beta}, \phi} & =\kappa \zeta_{3} \bar{\phi}^{\alpha \beta}+O\left(\kappa^{2}\right), \\
G^{\phi, \phi} & =\bar{\gamma}_{2}^{2} D(1+a D)+\kappa \zeta_{4} \phi+O\left(\kappa^{2}\right),
\end{aligned}
$$

where $\bar{\delta}_{\alpha \beta}^{\mu \nu}=\delta_{\alpha \beta}^{\mu \nu}-\frac{1}{D} g^{\mu \nu} g_{\alpha \beta}$ is the identity operator in the space of traceless symmetric rank-2 tensors, and the coefficients read

$\zeta_{1}=-2 \gamma_{1}\left(\gamma_{1}^{2}-2 \gamma_{3}\right)$,

$\zeta_{2}=\frac{D-4}{2} \gamma_{1}^{2} \bar{\gamma}_{2}+2 \gamma_{1} \bar{\gamma}_{5}$

$\zeta_{3}=2 \bar{\gamma}_{2}(1+a D)\left(\gamma_{3}+D \gamma_{4}\right)+\gamma_{1} \bar{\gamma}_{5}-\gamma_{1}^{2} \bar{\gamma}_{2}(2+a D)$,

$\zeta_{4}=\bar{\gamma}_{2} D(1+a D)\left(\frac{D-4}{2} \bar{\gamma}_{2}^{2}+4 \bar{\gamma}_{6}\right)$.

The inverse metric $\left(G^{-1}\right)_{A B}\left(A, B, \cdots=\bar{\phi}_{\mu \nu}, \phi\right)$ is given by

$$
\left(G^{-1}\right)_{A B}=\left(\begin{array}{cc}
\frac{1}{\gamma_{1}^{2}} \bar{\delta}_{\mu \nu, \alpha \beta} & 0 \\
0 & \frac{1}{\bar{\gamma}_{2}^{2} D(1+a D)}
\end{array}\right)+O(\kappa) .
$$

With these ingredients, we can proceed with the evaluation of the Christoffel symbols, whose nonzero components are

$$
\begin{aligned}
\Gamma_{\bar{\phi}_{\lambda \tau}, \bar{\phi}_{\alpha \beta}}^{\bar{\phi}_{\alpha \nu}} & =\frac{\kappa \zeta_{1}}{\gamma_{1}^{2}} g^{\mu \alpha} \bar{\delta}_{\lambda \tau}^{\beta \nu}+O\left(\kappa^{2}\right), \\
\Gamma_{\phi}^{\bar{\phi}_{\mu \nu}, \bar{\phi}_{\alpha \beta}} & =\kappa\left[\frac{2\left(\gamma_{3}+D \gamma_{4}\right)}{D \bar{\gamma}_{2}}-\frac{\gamma_{1}^{2}(4+D+4 a D)}{4 D(1+a D) \gamma_{2}}\right] \bar{\delta}^{\mu \nu, \alpha \beta}+O\left(\kappa^{2}\right), \\
\Gamma_{\bar{\phi}_{\lambda \tau}}^{\bar{\phi}_{\mu \nu}, \phi} & =\kappa\left(\frac{D-4}{4} \bar{\gamma}_{2}+\frac{\bar{\gamma}_{5}}{\gamma_{1}}\right) \bar{\delta}_{\lambda \tau}^{\mu \nu}+O\left(\kappa^{2}\right), \\
\Gamma_{\phi}^{\phi, \phi} & =\kappa\left(\frac{D-4}{4} \bar{\gamma}_{2}+\frac{2 \bar{\gamma}_{6}}{\bar{\gamma}_{2}}\right)+O\left(\kappa^{2}\right) .
\end{aligned}
$$

For the second covariant derivative of the action, we have

\footnotetext{
${ }^{3}$ Here, to avoid any kind of ambiguity, we made use of a more explicit notation for the indices.
} 


$$
\begin{aligned}
\left.\frac{\mathcal{D}^{2} S}{\delta \bar{\phi}_{\mu \nu} \delta \bar{\phi}_{\alpha \beta}}\right|_{\kappa \rightarrow 0} & =\gamma_{1}^{2}\left[g^{\beta \nu} \nabla^{\alpha} \nabla^{\mu}-\frac{1}{2} \bar{\delta}^{\mu \nu, \alpha \beta} \square-R^{\mu \alpha \nu \beta}-\frac{1}{4(1+a D)}\left(\frac{D-2}{2} R+D \Lambda\right) \bar{\delta}^{\mu \nu, \alpha \beta}\right], \\
\left.\frac{\mathcal{D}^{2} S}{\delta \bar{\phi}_{\mu \nu} \delta \phi}\right|_{\kappa \rightarrow 0} & =\gamma_{1} \bar{\gamma}_{2}\left(-\frac{D-2}{2} \nabla^{\mu} \nabla^{\nu}+\frac{D-4}{4} R^{\mu \nu}\right), \\
\left.\frac{\mathcal{D}^{2} S}{\delta \phi \delta \phi}\right|_{\kappa \rightarrow 0} & =\bar{\gamma}_{2}^{2}\left[\frac{(D-2)(D-1)}{2} \square-\frac{(D-4)(D-2)}{8} R-\frac{D^{2}}{4} \Lambda\right] .
\end{aligned}
$$

At this stage, it is clear that the dependence on the nonlinear quantum field parametrization was compensated by the Christoffel correction, just like in (45). In addition, the use of the parametrization in terms of the traceless and trace parts reveals that the improved bilinear operator can be written as constant matrix times a differential operator independent of $\gamma_{1}$ and $\bar{\gamma}_{2}$; thus, this dependence is trivial.

We point out that the conformal gauge fixing (68) does not require Faddeev-Popov ghosts because the conformal transformation has no derivatives [44]. Moreover, under the diffeomorphism (A1), the field $\sigma$ transforms as $\delta \sigma=-\nabla_{\mu} \sigma \xi^{\mu}$, and the terms in the ghost operator associated with the generators $R_{\mu}=-\nabla_{\mu} \sigma$ can be safely ignored at one-loop level since they produce third-order contributions in quantum field; as a consequence, we get (53). Therefore, even in the conformal parametrization, the final result matches the one presented in Eq. (60) once the conformal factor is identified with the trace of $\phi_{\mu \nu}$.

\section{CONCLUSIONS}

We performed the calculations of the one-loop divergences of the Vilkovisky unique effective action in quantum general relativity in an arbitrary, most general parametrization of quantum metric, including the conformal parametrization and the corresponding gauge fixing. Because of the similarity between the conformal parametrization and the two-dimensional quantum gravity, one could suspect that the unique effective action may lose its invariance and universality. We have shown that this does not happen and the one-loop divergences are universal. To achieve the positive result in the excessive conformal parametrization, the conformal gauge should be fixed before applying Vilkovisky's formalism, to guarantee the nondegeneracy of the field-space metric.

Finally, we fixed the dependence of the unique effective action on the arbitrary parameter $a$ of the term $g^{\mu \nu} g^{\alpha \beta}$ of the configuration-space metric $G^{\mu \nu, \alpha \beta}$ by the prescription that this metric is chosen as the metric contained in the highestderivative term of the bilinear form of the classical action in the minimal gauge. This choice is in consonance with the requirement that the metric in the space of the fields must be determined from the classical action, as proposed in the pioneer work [1]. We have shown that, although this term changes under modified parametrization of the quantum metric, the one-loop unique effective action does not change. This confirms the consistency of the mentioned additional requirement.

\section{ACKNOWLEDGMENTS}

The work of I. L. S. is partially supported by Conselho Nacional de Desenvolvimento Científico e TecnológicoCNPq under Grant No. 303635/2018-5.

\section{APPENDIX: GENERATORS OF GAUGE TRANSFORMATIONS}

The gauge generators for the field $\phi_{\mu \nu}$ have been evaluated in Ref. [9] up to the zeroth order in $\kappa$. Nonetheless, we need the expansion up to the next order. The reason is that the terms (16) and (17) depend on the covariant variational derivative of $R_{\mu \nu, \alpha}$ with respect to $\phi_{\mu \nu}$, requiring the $O(\kappa)$-approximation.

Consider the infinitesimal coordinate transformation

$$
x^{\mu} \mapsto x^{\prime \mu}=x^{\mu}+\xi^{\mu} .
$$

In the standard parametrization $g_{\mu \nu}^{\prime}$, the generator reads

$$
R_{\mu \nu, \gamma}^{\prime}\left(g^{\prime}\right)=-\left(g_{\mu \gamma}^{\prime} \nabla_{\nu}^{\prime}+g_{\nu \gamma}^{\prime} \nabla_{\mu}^{\prime}\right) .
$$

The generators of gauge transformation for the quantum field $\phi_{\mu \nu}$ can be obtained through a vector change of coordinates in the space of the field representations,

$$
R_{\mu \nu, \gamma}(\phi)=\frac{\partial\left(\kappa \phi_{\mu \nu}\right)}{\partial g_{\rho \sigma}^{\prime}} R_{\rho \sigma, \gamma}^{\prime}\left(g^{\prime}\right) .
$$

By using Eqs. (22), (A2), and (A3), it is possible to show that

$$
R_{\mu \nu, \gamma}(\phi)=R_{\mu \nu, \gamma}^{(0)}+\kappa R_{\mu \nu, \gamma}^{(1)}+O\left(\kappa^{2}\right)
$$

where

$R_{\mu \nu, \gamma}^{(0)}=-\frac{1}{\gamma_{1}}\left(g_{\mu \gamma} \nabla_{\nu}+g_{\nu \gamma} \nabla_{\mu}\right)+\frac{2 \gamma_{2}}{\gamma_{1}\left(\gamma_{1}+D \gamma_{2}\right)} g_{\mu \nu} \nabla_{\gamma}$

and 


$$
\begin{aligned}
R_{\mu \nu, \gamma}^{(1)}= & \left(r_{1}-1\right)\left(\phi_{\mu \gamma} \nabla_{\nu}+\phi_{\nu \gamma} \nabla_{\mu}\right)+r_{1}\left(g_{\mu \gamma} \phi_{\nu}^{\lambda}+g_{\nu \gamma} \phi_{\mu}^{\lambda}\right) \nabla_{\lambda}+r_{2} g_{\mu \nu} \phi_{\gamma}^{\lambda} \nabla_{\lambda}+r_{3} \phi_{\mu \nu} \nabla_{\gamma}-\left(\nabla_{\gamma} \phi_{\mu \nu}\right) \\
& +r_{4} \phi\left(g_{\mu \gamma} \nabla_{\nu}+g_{\nu \gamma} \nabla_{\mu}\right)+r_{5} g_{\mu \nu} \phi \nabla_{\gamma},
\end{aligned}
$$

with the coefficients

$$
\begin{aligned}
& r_{1}=\frac{\gamma_{3}}{\gamma_{1}^{3}}, \quad r_{2}=\frac{2 \gamma_{1}^{2} \gamma_{2}-4\left(\gamma_{2} \gamma_{3}-\gamma_{1} \gamma_{4}\right)}{\gamma_{1}^{2}\left(\gamma_{1}+D \gamma_{2}\right)}, \quad r_{3}=-\frac{2\left(2 \gamma_{2} \gamma_{3}-\gamma_{1} \gamma_{5}\right)}{\gamma_{1}^{2}\left(\gamma_{1}+D \gamma_{2}\right)}, \\
& r_{4}=\frac{\gamma_{5}-\gamma_{1} \gamma_{1}}{\gamma_{1}^{2}}, \quad r_{5}=\frac{2 \gamma_{1} \gamma_{2}^{2}+4 \gamma_{2}\left(\gamma_{2} \gamma_{3}-\gamma_{1} \gamma_{4}\right)-2 \gamma_{2} \gamma_{5}\left(3 \gamma_{1}+D \gamma_{2}\right)+4 \gamma_{1}^{2} \gamma_{6}}{\gamma_{1}^{2}\left(\gamma_{1}+D \gamma_{2}\right)} .
\end{aligned}
$$

The expressions (A5) and (A6) are sufficient for the one-loop calculations reported in the main part of the paper.

[1] G. A. Vilkovisky, The unique effective action in quantum field theory, Nucl. Phys. B234, 125 (1984); The Gospel according to DeWitt, in Quantum Theory of Gravity, edited by S. M. Christensen (Hilger, Bristol, 1984).

[2] B. S. DeWitt, The effective action, in Quantum Field Theory and Quantum Statistics, Essays in honor of the sixtieth birthday of E. S. Fradkin, Vol. 1: Quantum Statistics and methods of Field Theory, edited by C. J. Isham, I. A. Batalin, and G. A. Vilkovisky (Hilger, Bristol, 1987); The effective action, in Architecture of Fundamental Interactions at Short Distances, edited by P. Ramond and R. Stora (North-Holland, Amsterdam, 1987).

[3] G. 't Hooft and M. Veltman, One loop divergencies in the theory of gravitation, Ann. Inst. Henri Poincaré, A 20, 69 (1974), https://inspirehep.net/literature/95368.

[4] S. Deser and P. van Nieuwenhuisen, One-loop divergences of quantized Einstein-Maxwell fields, Phys. Rev. D 10, 401 (1974).

[5] R.E. Kallosh, O. V. Tarasov, and I. V. Tyutin, One-loop finiteness of quantum gravity off mass shell, Nucl. Phys. B137, 145 (1978).

[6] G. de Berredo-Peixoto, A. Penna-Firme, and I. L. Shapiro, One loop divergences of quantum gravity using conformal parametrization, Mod. Phys. Lett. A 15, 2335 (2000).

[7] M. Y. Kalmykov, Gauge and parametrization dependencies of the one loop counterterms in the Einstein gravity, Classical Quantum Gravity 12, 1401 (1995).

[8] M. Kalmykov, K. Kazakov, P. Pronin, and K. Stepanyantz, Detailed analysis of the dependence of the one loop counterterms on the gauge and parametrization in the Einstein gravity with the cosmological constant, Classical Quantum Gravity 15, 3777 (1998).

[9] J. D. Gonçalves, T. de Paula Netto, and I. L. Shapiro, Gauge and parametrization ambiguity in quantum gravity, Phys. Rev. D 97, 026015 (2018).

[10] A. T. Banin and I. L. Shapiro, Dilaton gravity in $d=2$ with trivial quantum corrections, JETP Lett. 58, 805 (1993), https://inspirehep.net/literature/365716; Gauge dependence and new kind of two-dimensional gravity theory with trivial quantum corrections, Phys. Lett. B 327, 17 (1994).

[11] S. D. Odintsov and I. L. Shapiro, One loop renormalization of two-dimensional induced quantum gravity, Phys. Lett. B 263, 183 (1991); Perturbative analysis of two-dimensional quantum gravity: Renormalization, gauge dependence and finiteness, Int. J. Mod. Phys. D 01, 571 (1992).

[12] D. Grumiller, W. Kummer, and D. V. Vassilevich, Dilaton gravity in two-dimensions, Phys. Rep. 369, 327 (2002).

[13] B. L. Giacchini, T. de Paula Netto, and I. L. Shapiro, On the Vilkovisky-DeWitt approach and renormalization group in effective quantum gravity, J. High Energy Phys. 10 (2020) 011.

[14] T. Taylor and G. Veneziano, Quantum gravity at large distances and the cosmological constant, Nucl. Phys. B345, 210 (1990).

[15] J. F. Barbero and J. Pérez-Mercader, Superspace dependence of the Vilkovisky-DeWitt effective action for quantum gravity, Phys. Rev. D 48, 3663 (1993).

[16] B. S. DeWitt, Dynamical Theory of Groups and Fields (Gordon and Breach, New York, 1965).

[17] A. O. Barvinsky and G. A. Vilkovisky, The generalized Schwinger-DeWitt technique and the unique effective action in quantum gravity, Phys. Lett. 131B, 313 (1983); The generalized Schwinger-Dewitt technique in gauge theories and quantum gravity, Phys. Rep. 119, 1 (1985).

[18] J. L. Synge, Relativity: The General Theory (North-Holland, Amsterdam, 1960).

[19] B. Voronov and I. Tyutin, Formulation of gauge theories of general form. II. Gauge invariant renormalizability and renormalization structure, Theor. Math. Phys. 52, 628 (1982).

[20] B. L. Voronov and I. V. Tyutin, On renormalization of $R^{2}$ gravitation, Yad. Fiz. 39, 998 (1984) [Sov. J. Nucl. Phys. 39, 998 (1984)], https://inspirehep.net/literature/208314.

[21] B. L. Voronov, P. M. Lavrov, and I. V. Tyutin, Canonical transformations and the gauge dependence in general gauge theories, Yad. Fiz. 36, 498 (1982) [Sov. J. Nucl. Phys. 36, 498 (1982)], https://inspirehep.net/literature/183748. 
[22] E. S. Fradkin and A. A. Tseytlin, On the new definition of off-shell Effective Action, Nucl. Phys. B234, 509 (1984).

[23] G. Kunstatter, Vilkovisky's unique effective action: An introduction and explicit calculation, in Super Field Theories, Proceedings of NATO Advanced Research Workshop on Superfield Theories, edited by H. C. Lee, V. Elias, G. Kunstatter, R. B. Mann, and K. S. Viswanathan, NATO ASI Series B Vol. 160 (Plenum, New York, 1987).

[24] S. R. Huggins, G. Kunstatter, H. P. Leivo, and D. J. Toms, The Vilkovisky-DeWitt effective action for quantum gravity, Nucl. Phys. B301, 627 (1988).

[25] P. Ellicott, D. Toms, and G. Kunstatter, Geometrical derivation of the Faddeev-Popov ansatz, Mod. Phys. Lett. A 04, 2397 (1989).

[26] L. Parker and D. Toms, Quantum Field Theory in Curved Spacetime: Quantized Fields and Gravity (Cambridge University Press, Cambridge, England, 2009).

[27] A. Rebhan, The Vilkovisky-DeWitt effective action and its application to Yang-Mills theories, Nucl. Phys. B288, 832 (1987).

[28] A. Rebhan, Feynman rules and $S$-matrix equivalence of the Vilkovisky-DeWitt effective action, Nucl. Phys. B298, 726 (1988).

[29] P. Ellicott and D. Toms, On the new effective action in quantum field theory, Nucl. Phys. B312, 700 (1989).

[30] B.S. DeWitt, Quantum theory of gravity. 2. The manifestly covariant theory, Phys. Rev. 162, 1195 (1967).

[31] I. G. Avramidi and A. O. Barvinsky, Asymptotic freedom in higher derivative quantum gravity, Phys. Lett. 159B, 269 (1985).

[32] I. G. Avramidi, Covariant methods for the calculation of the effective action in quantum field theory and investigation of higher-derivative quantum gravity, Ph.D. Thesis, Moscow University, 1986.

[33] H. T. Cho and R. Kantowski, The Unique One Loop Effective Action for the Six-Dimensional Einstein-Hilbert Action, Phys. Rev. Lett. 67, 422 (1991).

[34] S. Huggins, G. Kunstatter, H. Leivo, and D. Toms, On the Unique Effective Action in Five-dimensional Kaluza-Klein Theory, Phys. Rev. Lett. 58, 296 (1987).
[35] I. L. Buchbinder, P. M. Lavrov, and S. D. Odintsov, Unique effective action in Kaluza-Klein quantum theories and spontaneous compactification, Nucl. Phys. B308, 191 (1988); I. L. Buchbinder and S. D. Odintsov, Unique effective action in Kaluza-Klein theories and spontaneous compactification, Yad. Fiz. 47, 598 (1988) [Sov. J. Nucl. Phys. 47, 377 (1988)], https://inspirehep.net/literature/273565.

[36] I. L. Buchbinder, E. N. Kirillova, and S. D Odintsov, The Vilkovisky effective action in the even dimensional quantum gravity, Mod. Phys. Lett. A 04, 633 (1989); Vilkovisky's effective action in even dimensional theories of quantum gravitation, Sov. J. Nucl. Phys. 50, 168 (1989) [Yad. Fiz. 50, 269 (1989)], https://inspirehep.net/literature/ 286195.

[37] I. L. Buchbinder, V. P. Dergalev, and S. D. Odintsov, Vilkovisky-DeWitt effective action in multidimensional quantum gravity and antiperiodic boundary conditions, Theor. Math. Phys. 80, 776 (1989).

[38] N. Ohta, R. Percacci, and A. Pereira, Gauges and functional measures in quantum gravity I: Einstein theory, J. High Energy Phys. 06 (2016) 115; Gauges and functional measures in quantum gravity II: Higher derivative gravity, Eur. Phys. J. C 77, 611 (2017); $f\left(R, R_{\mu \nu}^{2}\right)$ at one loop, Phys. Rev. D 97, 104039 (2018).

[39] B. S. DeWitt, Quantum theory of gravity. 1. The canonical theory, Phys. Rev. 160, 1113 (1967).

[40] S. D. Odintsov, Does the Vilkovisky-De Witt effective action in quantum gravity depend on the configuration space metric?, Phys. Lett. B 262, 394 (1991).

[41] B. L. Giacchini, P. M. Lavrov, and I. L. Shapiro, Background field method and nonlinear gauges, Phys. Lett. B 797, 134882 (2019).

[42] S. Christensen and M. Duff, Quantizing gravity with a cosmological constant, Nucl. Phys. B170, 480 (1980).

[43] P. Lavrov, S. Odintsov, and I. Tyutin, On the unique effective action in field theory, Mod. Phys. Lett. A 03, 1273 (1988).

[44] E. S. Fradkin and A. A. Tseytlin, Renormalizable asymptotically free quantum theory of gravity, Nucl. Phys. B201, 469 (1982). 